\title{
Numerical Solution of First-Order Linear Differential Equations in Fuzzy Environment by Runge-Kutta-Fehlberg Method and Its Application
}

\author{
Sankar Prasad Mondal, ${ }^{1}$ Susmita Roy, ${ }^{1}$ and Biswajit Das ${ }^{2}$ \\ ${ }^{1}$ Department of Mathematics, National Institute of Technology, Agartala, Jirania, Tripura 799046, India \\ ${ }^{2}$ Department of Mechanical Engineering, National Institute of Technology, Agartala, Jirania, Tripura 799046, India \\ Correspondence should be addressed to Sankar Prasad Mondal; sankar.res07@gmail.com
}

Received 31 July 2015; Accepted 16 February 2016

Academic Editor: Yucheng Liu

Copyright (c) 2016 Sankar Prasad Mondal et al. This is an open access article distributed under the Creative Commons Attribution License, which permits unrestricted use, distribution, and reproduction in any medium, provided the original work is properly cited.

\begin{abstract}
The numerical algorithm for solving "first-order linear differential equation in fuzzy environment" is discussed. A scheme, namely, "Runge-Kutta-Fehlberg method," is described in detail for solving the said differential equation. The numerical solutions are compared with (i)-gH and (ii)-gH differential (exact solutions concepts) system. The method is also followed by complete error analysis. The method is illustrated by solving an example and an application.
\end{abstract}

\section{Introduction}

Fuzzy Differential Equation. In modeling of real natural phenomena, differential equations play an important role in many areas of discipline, exemplary in economics, biomathematics, science, and engineering. Many experts in such areas widely use differential equations in order to make some problems under study more comprehensible. In many cases, information about the physical phenomena related is always immanent with uncertainty.

Today, the study of differential equations with uncertainty is instantaneously growing as a new area in fuzzy analysis. The terms such as "fuzzy differential equation" and "fuzzy differential inclusion" are used interchangeably in mention to differential equations with fuzzy initial values or fuzzy boundary values or even differential equations dealing with functions on the space of fuzzy numbers. In the year 1987, the term "fuzzy differential equation (FDE)" was introduced by Kandel and Byatt [1]. There are different approaches to discuss the FDEs: (i) the Hukuhara derivative of a fuzzy number valued function is used, (ii) Hüllermeier [2] and Diamond and Watson [3-5] suggested a different formulation for the fuzzy initial value problems (FIVP) based on a family of differential inclusions, (iii) in $[6,7]$, Bede et al. defined generalized differentiability of the fuzzy number valued functions and studied FDE, and (iv) applying a parametric representation of fuzzy numbers, Chen et al. [8] established a new definition for the differentiation of a fuzzy valued function and used it in FDE.

Solution of Fuzzy Differential Equation by Numerical Techniques. Numerical methods are the methods by which we can find the solution of differential equation where the exact solution is critical to find. There exist various numerical methods for solving differential equation such as Setia et al. [9], Liu [10], and Setia et al. [11]. Our aim is to find the numerical techniques by which the solution of a linear or nonlinear first-order fuzzy differential equation comes easily and the solution is very close to the exact solution. There exist many techniques of numerical methods for finding the solution of fuzzy differential equation. Authors applied the method in certain types of fuzzy differential equation which shows that their techniques are best fit for that particular problem. The first paper on fuzzy differential equation and numerical analysis was published in 1999 by Ma et al. [12]. Allahviranloo et al. [13] apply the two-step method on fuzzy differential equations. Allahviranloo et al. [14] find the numerical solution by using predictor-corrector 
method. Allahviranloo et al. [15] find an algorithm for finding the solution Nth-order fuzzy linear differential equations using numerical techniques. Pederson and Sambandham in [16] use characterization theorem on hybrid fuzzy initial value problem. A soft computing technique, namely, artificial neural network, is implicated for solving FDE by Effati and Pakdaman [17]. Duraisamy and Usha [18] used modified Euler's method. The extension principle method was compared by Euler's method in Saberi Najafi et al. [19] article. Rostami et al. [20] find a numerical algorithm for solving nonlinear fuzzy differential equations. Moghadam and Dahaghin [21] apply two-step methods for numerical solution of FDE. Batiha in [22] finds an iterative solution of multispecies predator-prey model by variational iteration method. Ahmad and Hasan [23] proposed a new fuzzy version of Euler's method for solving differential equations with fuzzy initial value. Nirmala and Chenthur Pandian [24] give an idea for improving the numerical result on FDE. Shafiee et al. [25] use predictor-corrector method for nonlinear fuzzy Volterra integral equations. Comparison results on some numerical techniques on first-order fuzzy differential equation are illustrated by Ghanbari [26]. The use of variational iteration method for solving Nth-order fuzzy differential equations is shown by Jafari et al. [27]. Tapaswini and Chakraverty [28] discuss a new approach to fuzzy initial value problem by Improved Euler method. The solution of FIVP is compared by Least Square method and Adomian Decomposition method by Ahmed and Fadhel [29]. Solution of differential equation by Euler's method using fuzzy concept is developed by Saikia [30]. Ezzati et al. [31] find the numerical solution of Volterra-Fredholm integral equations with the help of inverse and direct discrete fuzzy transforms and collocation technique. The Adomian method is applied on second-order FDE by Wang and Guo [32]. Fard [33] uses iterative scheme to find the solution of generalized system of linear FDE, whereas Block method is used by Mehrkanoon et al. [34]. Asady and Alavi [35] apply a numerical method for solving $N$ th-order linear fuzzy differential equation.

Solution of Fuzzy Differential Equation by Runge-Kutta Method. Runge-Kutta method is well known for finding the approximate or numerical solution. In the last decade RungeKutta method is applied in fuzzy differential equation for finding the numerical solution. The researchers are giving various types of view to apply these methods. Someone changes the order and someone applies different types on FDE, a comparison of another method to Runge-Kutta method. The details of published work done in Runge-Kutta method are summarized below.

Numerical Solution of Fuzzy Differential Equations by Runge-Kutta method of order three is developed by Duraisamy and Usha [36]. Solution techniques for fourthorder Runge-Kutta method with higher order derivative approximations are developed by Nirmala and Chenthur Pandian [37]. Runge-Kutta method of order five is developed by Jayakumar et al. [38]. The techniques extended Runge-Kutta-like formulae of order four are developed by Ghazanfari and Shakerami [39]. Third-order Runge-Kutta method is developed by Kanagarajan and Sambath [40].
Runge-Kutta-Fehlberg method for hybrid fuzzy differential equation is solved by Jayakumar and Kanagarajan [41]. A different approach followed by Runge-Kutta method is applied by Akbarzadeh Ghanaie and Mohseni Moghadam [42]. "Numerical Solution of Fuzzy IVP with Trapezoidal and Triangular Fuzzy Numbers by Using Fifth-Order RungeKutta Method" is solved by Ghanbari [43]. "New MultiStep Runge-Kutta Method for Solving Fuzzy Differential Equations" is solved by Nirmala and Chenthur Pandian [44]. "Numerical Solution of Fuzzy Hybrid Differential Equation by Third Order Runge-Kutta Nystrom Method" is solved by Saveetha and Chenthur Pandian [45]. A new approach to solve fuzzy differential equation by using third-order RungeKutta method is developed by Deshmukh [46]. RungeKutta method of order four is developed by Duraisamy and Usha [47] and order five is developed by Jayakumar and Kanagarajan [48].

Application of Fuzzy Differential Equation. Fuzzy differential equations play a significant role in the fields of biology, engineering, and physics as well as among other fields of science, for example, in population models [49], civil engineering [50], bioinformatics and computational biology [51], quantum optics and gravity [52], modeling hydraulic [53], HIV model [54], decay model [55], predator-prey model [56], population dynamics model [57], friction model [58], growth model [59], bacteria culture model [60], bank account and drug concentration problem [61], barometric pressure problem [62], concentration problem [63], weight loss and oil production model [64], arm race model [65], vibration of mass [66], and fractional predator-prey equation [67].

Novelties. Although some developments are done, some new interest and new work have been done by ourselves which are mentioned below:

(i) Differential equation is solved in fuzzy environment by numerical techniques; that is, coefficients and initial condition both are taken as fuzzy number of a differential equation and solved by numerical techniques.

(ii) The numerical solution is compared with the exact solution ((i)-gH and (ii)-gH both cases).

(iii) Runge-Kutta-Fehlberg method for solving fuzzy differential equation is used.

(iv) For application purpose a mixture problem is considered.

(v) The solutions are found using different step length for better accuracy of the result.

(vi) The necessary algorithm for numerical solution is given.

Structure of the Paper. The paper is organized as follows: in Preliminary Concepts, the preliminary concepts and basic concepts on fuzzy number and fuzzy derivative are given. The method for finding the exact solution is discussed in Exact Solution of Fuzzy Differential Equation. In Numerical Solution of Fuzzy Differential Equation we proposed 
Runge-Kutta-Fehlberg method in fuzzy environment. The convergence of the said method and algorithm for finding the numerical results are also discussed in this section. Numerical Example shows a numerical example. In Application an important application, namely, mixture problem, is illustrated in fuzzy environment. Finally conclusions and future research scope of this paper are drawn in last section, Conclusion.

\section{Preliminary Concepts}

Definition 1 (fuzzy set). A fuzzy set $\widetilde{A}$ is defined by $\widetilde{A}=$ $\left\{\left(x, \mu_{\widetilde{A}}(x)\right): x \in A, \mu_{\widetilde{A}}(x) \in[0,1]\right\}$. In the pair $\left(x, \mu_{\widetilde{A}}(x)\right)$ the first element $x$ belongs to the classical set $A$ and the second element $\mu_{\widetilde{A}}(x)$ belongs to the interval $[0,1]$, called membership function.

Definition 2 ( $\alpha$-cut of a fuzzy set). The $\alpha$-level set (or interval of confidence at level $\alpha$ or $\alpha$-cut) of the fuzzy set $\widetilde{A}$ of $X$ is a crisp set $A_{\alpha}$ that contains all the elements of $X$ that have membership values in $A$ greater than or equal to $\alpha$; that is, $\widetilde{A}=\left\{x: \mu_{\widetilde{A}}(x) \geq \alpha, x \in X, 0<\alpha \leq 1\right\}$.

Definition 3 (fuzzy number). The basic definition of fuzzy number is as follows [30]: if we denote the set of all real numbers by $\mathscr{R}$ and the set of all fuzzy numbers on $\mathscr{R}$ is indicated by $\mathscr{R}_{\mathscr{F}}$ then a fuzzy number is mapping such that $u: \mathscr{R} \rightarrow[0,1]$, which satisfies the following four properties:

(i) $u$ is upper semicontinuous.

(ii) $u$ is a fuzzy convex; that is, $u(\lambda x+(1-\lambda) y) \geq$ $\min \{u(x), u(y)\}$ for all $x, y \in \mathscr{R}, \lambda \in[0,1]$.

(iii) $u$ is normal; that is, $\exists x_{0} \in \mathscr{R}$ for which $u\left(x_{0}\right)=1$.

(iv) supp $u=\{x \in \mathscr{R} \mid u(x)>0\}$ is support of $u$ and the closure of $(\operatorname{supp} u)$ is compact.

Definition 4 (parametric form of fuzzy number [31]). A fuzzy number is represented by an ordered pair of functions $\left(u_{1}(\alpha), u_{2}(\alpha)\right), 0 \leq \alpha \leq 1$, that satisfy the following condition:

(1) $u_{1}(\alpha)$ is a bounded left continuous nondecreasing function for any $\alpha \in[0,1]$.

(2) $u_{2}(\alpha)$ is a bounded left continuous nonincreasing function for any $\alpha \in[0,1]$.

(3) $u_{1}(\alpha) \leq u_{2}(\alpha)$ for any $\alpha \in[0,1]$.

Note. If $u_{1}(\alpha)=u_{2}(\alpha)=\alpha$, then $\alpha$ is a crisp number.

Definition 5 (generalized Hukuhara difference [20]). The generalized Hukuhara difference of two fuzzy numbers $u, v \in$ $\mathscr{R}_{\mathscr{F}}$ is defined as follows:

$$
u \ominus_{\mathrm{gH}} v=w \Longleftrightarrow\left\{\begin{aligned}
\text { (i) } u=v \oplus w, \\
\text { or } \quad \text { (ii) } v=u \oplus(-1) w .
\end{aligned}\right.
$$

Consider $[w]_{\alpha}=\left[w_{1}(\alpha), w_{1}(\alpha)\right]$; then $w_{1}(\alpha)=\min \left\{u_{1}(\alpha)-\right.$ $\left.v_{1}(\alpha), u_{2}(\alpha)-v_{2}(\alpha)\right\}$ and $w_{2}(\alpha)=\max \left\{u_{1}(\alpha)-v_{1}(\alpha), u_{2}(\alpha)-\right.$ $\left.v_{2}(\alpha)\right\}$
Here the parametric representation of a fuzzy valued function $f:[a, b] \rightarrow \mathscr{R}_{\mathscr{F}}$ is expressed by $[f(t)]_{\alpha}=$ $\left[f_{1}(t, \alpha), f_{2}(t, \alpha)\right], t \in[a, b], \alpha \in[0,1]$.

Definition 6 (generalized Hukuhara derivative for first order [20]). The generalized Hukuhara derivative of a fuzzy valued function $f:(a, b) \rightarrow \mathscr{R}_{\mathscr{F}}$ at $t_{0}$ is defined as

$$
f^{\prime}\left(t_{0}\right)=\lim _{h \rightarrow 0} \frac{f\left(t_{0}+h\right) \ominus_{\mathrm{gH}} f\left(t_{0}\right)}{h} .
$$

If $f^{\prime}\left(t_{0}\right) \in \mathscr{R}_{\mathscr{F}}$ satisfying (2) exists, we say that $f$ is generalized Hukuhara differentiable at $t_{0}$.

Also we say that $f(t)$ is (i)-gH differentiable at $t_{0}$ if

$$
\left[f^{\prime}\left(t_{0}\right)\right]_{\alpha}=\left[f_{1}^{\prime}\left(t_{0}, \alpha\right), f_{2}^{\prime}\left(t_{0}, \alpha\right)\right],
$$

and $f(t)$ is (ii)-gH differentiable at $t_{0}$ if

$$
\left[f^{\prime}\left(t_{0}\right)\right]_{\alpha}=\left[f_{2}^{\prime}\left(t_{0}, \alpha\right), f_{1}^{\prime}\left(t_{0}, \alpha\right)\right] .
$$

Definition 7 (see [6]). For arbitrary $u=\left(u_{1}, u_{2}\right)$ and $v=$ $\left(v_{1}, v_{2}\right) \in E^{1}$, the quantity

$$
D(u, v)=\left(\int_{0}^{1}\left(u_{1}-v_{1}\right)^{2}+\int_{0}^{1}\left(u_{2}-v_{2}\right)^{2}\right)^{1 / 2}
$$

is the distance between fuzzy numbers $u$ and $v$.

Definition 8 (triangular fuzzy number). A triangular fuzzy number (TFN) denoted by $\widetilde{A}$ is defined as $(a, b, c)$ where the membership function

$$
\mu_{\widetilde{A}}(x)= \begin{cases}0, & x \leq a, \\ \frac{x-a}{b-a}, & a \leq x \leq b, \\ 1, & x=b, \\ \frac{c-x}{c-b}, & b \leq x \leq c, \\ 0, & x \geq c .\end{cases}
$$

Definition $9(\alpha$-cut of a fuzzy set $\widetilde{A})$. The $\alpha$-cut of $\widetilde{A}=(a, b, c)$ is given by

$$
A_{\alpha}=[a+\alpha(b-a), c-\alpha(c-b)], \quad \forall \alpha \in[0,1] .
$$

Definition 10 (fuzzy ordinary differential equation (FODE)). Consider a simple 1st-order linear ordinary differential equation as follows:

$$
\frac{d x}{d t}=k x+x_{0} \quad \text { with initial condition } x\left(t_{0}\right)=\gamma .
$$

The above ordinary differential equation is called fuzzy ordinary differential equation if any one of the following three cases holds:

(i) Only $\gamma$ is a fuzzy number (Type-I).

(ii) Only $k$ is a fuzzy number (Type-II).

(iii) Both $k$ and $\gamma$ are fuzzy numbers (Type-III). 


\section{Exact Solution of Fuzzy Differential Equation}

Consider the fuzzy initial value problem

$$
y^{\prime}(t)=f(t, y(t)), \quad t \in I=[0, T] \text { with } y(0)=y_{0},
$$

where $f$ is a continuous mapping from $R_{+} \times R$ into $R$ and $y_{0} \in E$ with $r$-level sets

$$
\left[y_{0}\right]_{r}=\left[y_{1}(0 ; \alpha), y_{2}(0 ; \alpha)\right], \quad \alpha \in(0,1] .
$$

We write $f(t, y)=\left[f_{1}(t, y), f_{2}(t, y)\right]$ and $f_{1}(t, y)=$ $F\left[t, y_{1}, y_{2}\right], f_{2}(t, y)=G\left[t, y_{1}, y_{2}\right]$.

Because of $y^{\prime}(t)=f(t, y)$ we have the following.

When $y(t, y)$ is (i)-gH differentiable

$$
\begin{aligned}
& y_{1}^{\prime}(t, \alpha)=F\left[t ; y_{1}(t ; \alpha), y_{2}(t ; \alpha)\right], \\
& y_{2}^{\prime}(t, \alpha)=G\left[t ; y_{1}(t ; \alpha), y_{2}(t ; \alpha)\right] .
\end{aligned}
$$

When $y(t, y)$ is (ii)-gH differentiable

$$
\begin{aligned}
& y_{2}^{\prime}(t, \alpha)=F\left[t ; y_{1}(t ; \alpha), y_{2}(t ; \alpha)\right], \\
& y_{1}^{\prime}(t, \alpha)=G\left[t ; y_{1}(t ; \alpha), y_{2}(t ; \alpha)\right],
\end{aligned}
$$

where, by using extension principle, we have the membership function

$$
f(t ; y(t))(s)=\operatorname{Sup}\{y(t)(\tau) \backslash s=f(t, \tau)\}, s \in R .
$$

So fuzzy number is $f(t ; y(t))$. From this it follows that

$$
\begin{array}{r}
{[f(t ; y(t))]_{\alpha}=\left[f_{1}(t, y(t) ; \alpha), f_{2}(t, y(t) ; \alpha)\right],} \\
\alpha \in[0 ; 1],
\end{array}
$$

where

$$
\begin{aligned}
& f_{1}(t, y(t) ; \alpha)=F\left[t ; y_{1}(t ; \alpha), y_{2}(t ; \alpha)\right] \\
& \quad=\min \left\{f(t, u) \backslash u \in\left[y_{1}(t ; \alpha), y_{2}(t ; \alpha)\right]\right\}, \\
& f_{2}(t, y(t) ; \alpha)=G\left[t ; y_{1}(t ; \alpha), y_{2}(t ; \alpha)\right] \\
& \quad=\max \left\{f(t, u) \backslash u \in\left[y_{1}(t ; \alpha), y_{2}(t ; \alpha)\right]\right\} .
\end{aligned}
$$

Note. (1) Both cases ((i)-gH and (ii)-gH) can be applied to a FDE for finding exact solution.

(2) After taking $\alpha$-cut of the given FDE, it transforms to system of ordinary differential equation.

\section{Numerical Solution of Fuzzy Differential Equation}

4.1. Runge-Kutta-Fehlberg Method for Ordinary (Crisp) Differential Equation. Consider the initial value problem $y^{\prime}(t)=$ $f(t, y(t)) ; y\left(t_{0}\right)=y_{0}$.

The Runge-Kutta-Fehlberg method (denoted as RKF45) is one way to try to resolve this problem.
The problem is to solve the initial value problem in above equation by means of Runge-Kutta methods of order 4 and order 5 .

First we need some definitions:

$$
\begin{aligned}
k_{1} & =h f\left(t_{i}, y_{i}\right), \\
k_{2} & =h f\left(t_{i}+\frac{1}{4} h, y_{i}+\frac{1}{4} k_{1}\right), \\
k_{3} & =h f\left(t_{i}+\frac{3}{8} h, y_{i}+\frac{3}{32} k_{1}+\frac{9}{32} k_{2}\right), \\
k_{4} & =h f\left(t_{i}+\frac{12}{13} h, y_{i}+\frac{1932}{2197} k_{1}-\frac{7200}{2197} k_{2}\right. \\
& \left.+\frac{7296}{2197} k_{3}\right), \\
k_{5} & =h f\left(t_{i}+h, y_{i}+\frac{439}{216} k_{1}-8 k_{2}+\frac{3680}{513} k_{3}\right. \\
& \left.-\frac{845}{4104} k_{4}\right), \\
k_{6} & =h f\left(t_{i}+h, y_{i}-\frac{8}{27} k_{1}+2 k_{2}-\frac{3544}{2565} k_{3}+\frac{1859}{4104} k_{4}\right. \\
& \left.-\frac{11}{40} k_{5}\right) .
\end{aligned}
$$

Then an approximation to the solution of initial value problem is made using Runge-Kutta method of order 4:

$$
y_{i+1}=y_{i}+\frac{25}{216} k_{1}+\frac{1408}{2565} k_{3}+\frac{2197}{4101} k_{4}-\frac{1}{5} k_{5} \text {. }
$$

A better value for the solution is determined using a RungeKutta method of order 5:

$$
\begin{aligned}
z_{i+1}= & y_{i}+\frac{16}{135} k_{1}+\frac{6656}{12,825} k_{3}+\frac{28,561}{56,430} k_{4}-\frac{9}{50} k_{5} \\
& +\frac{2}{55} k_{6} .
\end{aligned}
$$

The optimal step size $s h$ can be determined by multiplying the scalar $s$ times the step size $h$. The scalar $s$ is

$$
\begin{aligned}
s & =\left(\frac{\epsilon h}{2\left|z_{i+1}-y_{i+1}\right|}\right)^{1 / 4} \\
& =0.0840896\left(\frac{\epsilon h}{\left|z_{i+1}-y_{i+1}\right|}\right)^{1 / 4},
\end{aligned}
$$

where $\epsilon$ is the specified error control tolerance.

Note that RK4 requires 4 function evaluations and RK5 requires 6 evaluations, that is, 10 for RK4 and RK5. Fehlberg devised a method to get RK4 and RK5 results using only 6 function evaluations by using some of $K$ values in both methods.

4.2. Runge-Kutta-Fehlberg Method for Solving Fuzzy Differential Equations. Let $Y=\left[Y_{1}, Y_{2}\right]$ be the exact solution and let 
$y=\left[y_{1}, y_{2}\right]$ be the approximated solution of the fuzzy initial value problem. $\alpha)$.

Let $[Y(t)]_{\alpha}=\left[Y_{1}(t, \alpha), Y_{2}(t, \alpha)\right],[y(t)]_{r}=\left[y_{1}(t, \alpha), y_{2}(t\right.$,

Throughout this argument, the value of $r$ is fixed. Then the exact and approximated solution at $t_{n}$ are, respectively, denoted by

$$
\begin{aligned}
& {\left[Y\left(t_{n}\right)\right]_{\alpha}=\left[Y_{1}\left(t_{n}, \alpha\right), Y_{2}\left(t_{n}, \alpha\right)\right],} \\
& {\left[y\left(t_{n}\right)\right]_{\alpha}=\left[y_{1}\left(t_{n}, \alpha\right), y_{2}\left(t_{n}, \alpha\right)\right] .}
\end{aligned}
$$

The grid points at which the solution is calculated are $h=$ $\left(T-t_{0}\right) / N, t_{i}=t_{0}+i h, 0 \leq i \leq N$.

Then we obtained

$$
\begin{aligned}
y_{1}\left(t_{n+1}, \alpha\right)= & y_{1}\left(t_{n}, \alpha\right)+\frac{16}{135} K_{1}+\frac{6656}{12,825} K_{3} \\
& +\frac{28,561}{56,430} K_{4}-\frac{9}{50} K_{5}+\frac{2}{55} K_{6},
\end{aligned}
$$

where

$$
\begin{aligned}
& K_{1}=h F\left(t_{n}, y_{1}\left(t_{n}, \alpha\right), y_{2}\left(t_{n}, \alpha\right)\right) \text {, } \\
& K_{2}=h F\left(t_{n}+\frac{1}{4} h, y_{1}\left(t_{n}, \alpha\right)+\frac{1}{4} K_{1}, y_{2}\left(t_{n}, \alpha\right)\right. \\
& \left.+\frac{1}{4} K_{1}\right) \\
& K_{3}=h F\left(t_{n}+\frac{3}{8} h, y_{1}\left(t_{n}, \alpha\right)+\frac{3}{32} K_{1}\right. \\
& \left.+\frac{9}{32} K_{2}, y_{2}\left(t_{n}, \alpha\right)+\frac{3}{32} K_{1}+\frac{9}{32} K_{2}\right) \text {, } \\
& K_{4}=h F\left(t_{n}+\frac{12}{13} h, y_{1}\left(t_{n}, \alpha\right)+\frac{1932}{2197} K_{1}-\frac{7200}{2197} K_{2}\right. \\
& +\frac{7296}{2197} K_{3}, y_{2}\left(t_{n}, \alpha\right)+\frac{1932}{2197} K_{1}-\frac{7200}{2197} K_{2} \\
& \left.+\frac{7296}{2197} K_{3}\right) \\
& K_{5}=h F\left(t_{n}+h, y_{1}\left(t_{n}, \alpha\right)+\frac{439}{216} K_{1}-8 K_{2}\right. \\
& +\frac{3680}{513} K_{3}-\frac{845}{4104} K_{4}, y_{2}\left(t_{n}, \alpha\right)+\frac{439}{216} K_{1}-8 K_{2} \\
& \left.+\frac{3680}{513} K_{3}-\frac{845}{4104} K_{4}\right) \text {, } \\
& K_{6}=h F\left(t_{n}+h, y_{1}\left(t_{n}, \alpha\right)-\frac{8}{27} K_{1}+2 K_{2}-\frac{3544}{2565} K_{3}\right. \\
& +\frac{1859}{4104} K_{4}-\frac{11}{40} K_{5}, y_{2}\left(t_{n}, \alpha\right)-\frac{8}{27} K_{1}+2 K_{2} \\
& \left.-\frac{3544}{2565} K_{3}+\frac{1859}{4104} K_{4}-\frac{11}{40} K_{5}\right) \text {, }
\end{aligned}
$$

$$
\begin{gathered}
y_{2}\left(t_{n+1}, \alpha\right)=y_{2}\left(t_{n}, \alpha\right)+\frac{16}{135} L_{1}+\frac{6656}{12,825} L_{3} \\
+\frac{28,561}{56,430} L_{4}-\frac{9}{50} L_{5}+\frac{2}{55} L_{6},
\end{gathered}
$$

where

$$
\begin{aligned}
L_{1} & =h G\left(t_{n}, y_{1}\left(t_{n}, \alpha\right), y_{2}\left(t_{n}, \alpha\right)\right), \\
L_{2} & =h G\left(t_{n}+\frac{1}{4} h, y_{1}\left(t_{n}, \alpha\right)+\frac{1}{4} L_{1}, y_{2}\left(t_{n}, \alpha\right)\right. \\
& \left.+\frac{1}{4} L_{1}\right), \\
L_{3} & =h G\left(t_{n}+\frac{3}{8} h, y_{1}\left(t_{n}, \alpha\right)+\frac{3}{32} L_{1}\right. \\
& \left.+\frac{9}{32} L_{2}, y_{2}\left(t_{n}, \alpha\right)+\frac{3}{32} L_{1}+\frac{9}{32} L_{2}\right), \\
L_{4} & =h G\left(t_{n}+\frac{12}{13} h, y_{1}\left(t_{n}, \alpha\right)+\frac{1932}{2197} L_{1}-\frac{7200}{2197} L_{2}\right. \\
& +\frac{7296}{2197} L_{3}, y_{2}\left(t_{n}, \alpha\right)+\frac{1932}{2197} L_{1}-\frac{7200}{2197} L_{2} \\
& \left.+\frac{7296}{2197} L_{3}\right), \\
& \left.-\frac{3544}{2565} L_{3}+\frac{1859}{4104} L_{4}-\frac{11}{40} L_{5}\right) \cdot \\
L_{6} & =h G\left(t_{n}+h, y_{1}\left(t_{n}, \alpha\right)-\frac{8}{27} L_{1}+2 L_{2}-\frac{3544}{2565} L_{3}\right. \\
& \left.-\frac{845}{4104} L_{4}\right), y_{1}\left(t_{n}, \alpha\right)+\frac{439}{216} L_{1}-8 L_{2}+\frac{3680}{513} L_{3} \\
& =\frac{11}{40} L_{5}, y_{2}\left(t_{n}, \alpha\right)-\frac{8}{27} L_{1}+2 L_{2} \\
& \frac{839}{216} L_{1}-8 L_{2}+\frac{3680}{513} L_{3}
\end{aligned}
$$

4.3. Convergence of Fuzzy Runge-Kutta-Fehlberg Method. The solution is calculated by grid points at $a=t_{0} \leq t_{1} \leq \cdots \leq$ $t_{N}=b$ and $h=(b-a) / N=t_{n+1}-t_{n}$.

Therefore, we have

$$
\begin{aligned}
Y_{1}\left(t_{n+1}, \alpha\right)= & Y_{1}\left(t_{n}, \alpha\right)+F\left(t_{n}, Y_{1}\left(t_{n}, \alpha\right), Y_{2}\left(t_{n}, \alpha\right)\right), \\
Y_{2}\left(t_{n+1}, \alpha\right)= & Y_{2}\left(t_{n}, \alpha\right) \\
& +G\left(t_{n}, Y_{1}\left(t_{n}, \alpha\right), Y_{2}\left(t_{n}, \alpha\right)\right), \\
y_{1}\left(t_{n+1}, \alpha\right)= & y_{1}\left(t_{n}, \alpha\right)+F\left(t_{n}, y_{1}\left(t_{n}, \alpha\right), y_{2}\left(t_{n}, \alpha\right)\right), \\
y_{2}\left(t_{n+1}, \alpha\right)= & y_{2}\left(t_{n}, \alpha\right)+G\left(t_{n}, y_{1}\left(t_{n}, \alpha\right), y_{2}\left(t_{n}, \alpha\right)\right) .
\end{aligned}
$$


Clearly, $y_{1}(t, \alpha)$ and $y_{2}(t, \alpha)$ converge to $Y_{1}(t, \alpha)$ and $Y_{2}(t, \alpha)$, respectively, whenever $h \rightarrow 0$; that is,

$$
\begin{aligned}
& \lim _{h \rightarrow 0} y_{1}(t, \alpha)=Y_{1}(t, \alpha), \\
& \lim _{h \rightarrow 0} y_{2}(t, \alpha)=Y_{2}(t, \alpha) .
\end{aligned}
$$

Proof. Before we go to the main proof we need to know some results.

Lemma 11. Let the sequence of numbers $\{W\}_{n=0}^{N}$ satisfy

$$
\left|W_{n+1}\right| \leq A\left|W_{n}\right|+B, \quad 0 \leq n \leq N-1
$$

for some given positive constants $A$ and $B$. Then

$$
\left|W_{n}\right| \leq A^{n}\left|W_{0}\right|+B \frac{A^{n}-1}{A-1}, \quad 0 \leq n \leq N .
$$

Lemma 12. Let the sequence of numbers $\{W\}_{n=0}^{N}$ and $\{V\}_{n=0}^{N}$ satisfy

$$
\begin{gathered}
\left|W_{n+1}\right| \leq\left|W_{n}\right|+A \max \left\{\left|W_{n}\right|,\left|V_{n}\right|\right\}+B, \\
\left|V_{n+1}\right| \leq\left|V_{n}\right|+A \max \left\{\left|W_{n}\right|,\left|V_{n}\right|\right\}+B,
\end{gathered}
$$

for some given positive constants $A$ and $B$, and denote

$$
U_{n}=\left|W_{n}\right|+\left|V_{n}\right|, \quad 0 \leq n \leq N .
$$

Then

$$
U_{n} \leq \bar{A}^{n} U_{0}+\bar{B} \frac{\bar{A}^{n}-1}{\bar{A}-1}, \quad 0 \leq n \leq N,
$$

where $\bar{A}=1+2 A$ and $\bar{B}=2 B$.

Let $F(t, u, v)$ and $G(t, u, v)$ be obtained by substituting $\left[y_{1}(t, \alpha), y_{2}(t, \alpha)\right]=[u, v]$ in $(21)$ and (23); that is,

$$
\begin{aligned}
F(t, u, v)= & \frac{16}{135} K_{1}(t, u, v)+\frac{6656}{12,825} K_{3}(t, u, v) \\
& +\frac{28,561}{56,430} K_{4}(t, u, v)-\frac{9}{50} K_{5}(t, u, v) \\
& +\frac{2}{55} K_{6}(t, u, v), \\
G(t, u, v)= & \frac{16}{135} L_{1}(t, u, v)+\frac{6656}{12,825} L_{3}(t, u, v) \\
& +\frac{28,561}{56,430} L_{4}(t, u, v)-\frac{9}{50} L_{5}(t, u, v) \\
& +\frac{2}{55} L_{6}(t, u, v) .
\end{aligned}
$$

The domain where $F$ and $G$ are defined is as

$$
\begin{aligned}
H & =\{(t, u, v) \mid 0 \leq t \leq T,-\infty<v<\infty,-\infty<u \\
& \leq v\} .
\end{aligned}
$$

Theorem 13. Let $F(t, u, v)$ and $G(t, u, v)$ belong to $C^{p-1}(K)$ and let the partial derivative of $F$ and $G$ be bounded over $K$. Then for arbitrary fixed $0 \leq \alpha \leq 1$, the numerical solution of (9), $\left[y_{1}(t, \alpha), y_{2}(t, \alpha)\right]$ converges to the exact solution $\left[Y_{1}(t, \alpha), Y_{2}(t, \alpha)\right]$.

Proof (see [46]). By using Taylor's theorem we get

$$
\begin{aligned}
Y_{1}\left(t_{n+1}, \alpha\right)= & Y_{1}\left(t_{n}, \alpha\right) \\
& +h F\left(t_{n}, Y_{1}\left(t_{n}, \alpha\right), Y_{2}\left(t_{n}, \alpha\right)\right) \\
& +\frac{h^{p+1}}{(p+1) !} Y_{1}^{(p+1)}\left(\xi_{n, 1}\right), \\
Y_{2}\left(t_{n+1}, \alpha\right)= & Y_{2}\left(t_{n}, \alpha\right) \\
& +h G\left(t_{n}, Y_{1}\left(t_{n}, \alpha\right), Y_{2}\left(t_{n}, \alpha\right)\right) \\
& +\frac{h^{p+1}}{(p+1) !} Y_{2}^{(p+1)}\left(\xi_{n, 2}\right),
\end{aligned}
$$

where $\xi_{n, 1}, \xi_{n, 2} \in\left(t_{n}, t_{n+1}\right)$.

Now if we denote

$$
\begin{gathered}
W_{n}=Y_{1}\left(t_{n}, \alpha\right)-y_{1}\left(t_{n}, \alpha\right), \\
V_{n}=Y_{2}\left(t_{n}, \alpha\right)-y_{2}\left(t_{n}, \alpha\right),
\end{gathered}
$$

then the above two expressions converted to

$$
\begin{aligned}
& W_{n+1}=W_{n}+h\left\{F\left(t_{n}, Y_{1}\left(t_{n}, \alpha\right), Y_{2}\left(t_{n}, \alpha\right)\right)\right. \\
& \left.-F\left(t_{n}, y_{1}\left(t_{n}, \alpha\right), y_{2}\left(t_{n}, \alpha\right)\right)\right\}+\frac{h^{p+1}}{(p+1) !} \\
& \cdot Y_{1}^{(p+1)}\left(\xi_{n, 1}\right), \\
& V_{n+1}=V_{n}+h\left\{G\left(t_{n}, Y_{1}\left(t_{n}, \alpha\right), Y_{2}\left(t_{n}, \alpha\right)\right)\right. \\
& \left.-G\left(t_{n}, y_{1}\left(t_{n}, \alpha\right), y_{2}\left(t_{n}, \alpha\right)\right)\right\}+\frac{h^{p+1}}{(p+1) !} \\
& \cdot Y_{2}^{(p+1)}\left(\xi_{n, 2}\right) .
\end{aligned}
$$

Hence we can write

$$
\begin{aligned}
& \left|W_{n+1}\right| \leq\left|W_{n}\right|+2 L h \max \left\{\left|W_{n}\right|,\left|V_{n}\right|\right\}+\frac{h^{p+1}}{(p+1) !} M, \\
& \left|V_{n+1}\right| \leq\left|V_{n}\right|+2 L h \max \left\{\left|W_{n}\right|,\left|V_{n}\right|\right\}+\frac{h^{p+1}}{(p+1) !} M,
\end{aligned}
$$

where $M=\max \left\{\max \left|Y_{1}^{(p+1)}(t ; \alpha)\right|, \max \left|Y_{2}^{(p+1)}(t ; \alpha)\right|\right\}$ for $t \epsilon$ $[0, T]$ and $L>0$ is a bound for the partial derivative of $F$ and G.

Therefore we can write

$$
\begin{gathered}
\left|W_{n}\right| \leq(1+4 L h)^{n}\left|U_{0}\right|+\frac{2 h^{p+1}}{(p+1) !} M \frac{(1+4 L h)^{n}-1}{4 L h}, \\
\left|V_{n}\right| \leq(1+4 L h)^{n}\left|U_{0}\right|+\frac{2 h^{p+1}}{(p+1) !} M \frac{(1+4 L h)^{n}-1}{4 L h},
\end{gathered}
$$

where $\left|U_{0}\right|=\left|W_{0}\right|+\left|V_{0}\right|$. 
In particular,

$$
\begin{aligned}
\left|W_{N}\right| \leq & (1+4 L h)^{N}\left|U_{0}\right| \\
& +\frac{2 h^{p+1}}{(p+1) !} M \frac{(1+4 L h)^{T / h}-1}{4 L h} \\
\left|V_{N}\right| \leq & (1+4 L h)^{N}\left|U_{0}\right| \\
& +\frac{2 h^{p+1}}{(p+1) !} M \frac{(1+4 L h)^{T / h}-1}{4 L h} .
\end{aligned}
$$

Since $W_{0}=V_{0}=0$, we have

$$
\begin{aligned}
& \left|W_{N}\right| \leq M \frac{e^{4 L T}-1}{2 L(p+1) !} h^{p}, \\
& \left|V_{N}\right| \leq M \frac{e^{4 L T}-1}{2 L(p+1) !} h^{p} .
\end{aligned}
$$

Thus, if $h \rightarrow 0$, we get $W_{N} \rightarrow 0$ and $V_{N} \rightarrow 0$, which completes the proof.

\subsection{Algorithm for Finding the Numerical Solution}

Step 1. $F\left(t, y_{1}, y_{2}\right) \leftarrow$ "Function to be supplied"

$G\left(t, y_{1}, y_{2}\right) \leftarrow$ "Function to be supplied"

Step 2. Read $t(0), y_{1}(0), y_{2}(0), h$, limit.

Step 3. For $i=0(1)$ limit

$$
\begin{aligned}
& K_{1} \leftarrow h F\left(t_{i}, y_{1}\left(t_{i}, r\right), y_{2}\left(t_{i}, r\right)\right) \\
& K_{2} \leftarrow h \cdot F\left(t_{i}+(1 / 4) h, y_{1}\left(t_{i}, r\right)+(1 / 4) K_{1}, y_{2}\left(t_{i}, r\right)+\right. \\
& \left.(1 / 4) K_{1}\right) \\
& K_{3} \leftarrow h \cdot F\left(t_{i}+(3 / 8) h, y_{1}\left(t_{i}, r\right)+(3 / 32) K_{1}+(9 / 32) K_{2},\right. \\
& \left.y_{2}\left(t_{i}, r\right)+(3 / 32) K_{1}+(9 / 32) K_{2}\right) \\
& K_{4} \leftarrow h \cdot F\left(t_{i}+(12 / 13) h, y_{1}\left(t_{i}, r\right)+(1932 / 2197) K_{1}-\right. \\
& (7200 / 2197) K_{2}+(7296 / 2197) K_{3}, y_{2}\left(t_{i}, r\right)+(1932 / \\
& \left.2197) K_{1}-(7200 / 2197) K_{2}+(7296 / 2197) K_{3}\right) \\
& K_{5} \leftarrow h F\left(t_{i}+h, y_{1}\left(t_{i}, r\right)+(439 / 216) K_{1}-8 K_{2}+(3680 /\right. \\
& 513) K_{3}-(845 / 4104) K_{4}, y_{2}\left(t_{i}, r\right)+(439 / 216) K_{1}- \\
& \left.8 K_{2}+(3680 / 513) K_{3}-(845 / 4104) K_{4}\right) \\
& K_{6} \leftarrow h F\left(t_{i}+h, y_{1}\left(t_{i}, r\right)-(8 / 27) K_{1}+2 K_{2}-(3544 /\right. \\
& 2565) K_{3}+(1859 / 4104) K_{4}-(11 / 40) K_{5}, y_{2}\left(t_{i}, r\right)- \\
& (8 / 27) K_{1}+2 K_{2}-(3544 / 2565) K_{3}+(1859 / 4104) K_{4}- \\
& \left.(11 / 40) K_{5}\right) \\
& L_{1} \leftarrow h \cdot G\left(t_{i}, y_{1}\left(t_{i}, r\right), y_{2}\left(t_{i}, r\right)\right) \\
& L_{2} \leftarrow h G\left(t_{i}+(1 / 4) h, y_{1}\left(t_{i}, r\right)+(1 / 4) L_{1}, y_{2}\left(t_{i}, r\right)+\right. \\
& \left.(1 / 4) L_{1}\right) \\
& L_{3} \leftarrow h G\left(t_{i}+(3 / 8) h, y_{1}\left(t_{i}, r\right)+(3 / 32) L_{1}+(9 / 32) L_{2},\right. \\
& \left.y_{2}\left(t_{i}, r\right)+(3 / 32) L_{1}+(9 / 32) L_{2}\right) \\
& L_{4} \leftarrow h G\left(t_{i}+(12 / 13) h, y_{1}\left(t_{i}, r\right)+(1932 / 2197) L_{1}-\right. \\
& (7200 / 2197) L_{2}+(7296 / 2197) L_{3}, y_{2}\left(t_{i}, r\right)+(1932 / \\
& \left.2197) L_{1}-(7200 / 2197) L_{2}+(7296 / 2197) L_{3}\right)
\end{aligned}
$$

$$
\begin{aligned}
& L_{5} \leftarrow h G\left(t_{i}+h, y_{1}\left(t_{i}, r\right)+(439 / 216) L_{1}-8 L_{2}+\right. \\
& (3680 / 513) L_{3}-(845 / 4104) L_{4}, y_{2}\left(t_{i}, r\right)+(439 / \\
& \left.216) L_{1}-8 L_{2}+(3680 / 513) L_{3}-(845 / 4104) L_{4}\right) \\
& L_{6} \leftarrow h G\left(t_{i}+h, y_{1}\left(t_{i}, r\right)-(8 / 27) L_{1}+2 L_{2}-\right. \\
& (3544 / 2565) L_{3}+(1859 / 4104) L_{4}-(11 / 40) L_{5}, y_{2}\left(t_{i},\right. \\
& r)-(8 / 27) L_{1}+2 L_{2}-(3544 / 2565) L_{3}+(1859 / \\
& \left.4104) L_{4}-(11 / 40) L_{5}\right)
\end{aligned}
$$

Step 4.

$y_{1}\left(t_{i+1}, r\right)=y_{1}\left(t_{i}, r\right)+(16 / 135) K_{1}+(6656 / 12,825) K_{3}+$ $(28,561 / 56,430) K_{4}-(9 / 50) K_{5}+(2 / 55) K_{6}$

Step 5.

$$
y_{2}\left(t_{i+1}, r\right)=y_{2}\left(t_{i}, r\right)+(16 / 135) L_{1}+(6656 / 12,825) L_{3}+
$$$$
(28,561 / 56,430) L_{4}-(9 / 50) L_{5}+(2 / 55) L_{6}
$$

Step 6. $t_{i+1}=t_{i}+h$. Write $y_{1}\left(t_{i+1}, r\right), y_{2}\left(t_{i+1}, r\right), t_{i+1}$.

Step 7. Next $i$

Step 8. End.

\section{Numerical Example}

Example. Solve $y^{\prime}=-y+t+1$ with initial condition $y(0)=$ $(0.96,1,1.01)$. Then find the solution at $t=0.1$.

Solution. For (i)-gH differentiable case the exact solution is

$$
\begin{aligned}
& y_{1}(r, t)=t+(0.96+0.04 r) e^{-t} \\
& y_{2}(r, t)=t+(1.01-0.01 r) e^{-t}
\end{aligned}
$$

and for (ii)-gH differentiable case the exact solution is

$$
\begin{aligned}
& y_{1}(r, t)=1+t+(-0.04+0.04 r) e^{t} \\
& y_{2}(r, t)=1+t+(0.01-0.01 r) e^{t}
\end{aligned}
$$

Remark 14. From Figure 1 and Table 1 we conclude that the lower exact solution ((i)-gH case) is approximately equal to the numerical solution when we take the step length $h=$ 0.01 (for $h=0.001$ is nearly equal), whereas the lower exact solution ((ii)-gH case) is approximately equal to the numerical solution when we take the step length $h=0.1$.

Remark 15. From Figure 2 and Table 1 we conclude that the upper exact solution ((i)-gH case) is approximately equal to the numerical solution when we take the step length $h=$ 0.001 (for $h=0.01$ is nearly equal), whereas the upper exact solution ((ii)-gH case) is approximately equal to the numerical solution when we take the step length $h=0.1$.

\section{Application}

Problem. A tank initially contains 300 gals of brine which has dissolved in $c$ lbs of salt. Coming into the tank at 3 gals $/ \mathrm{min}$ 


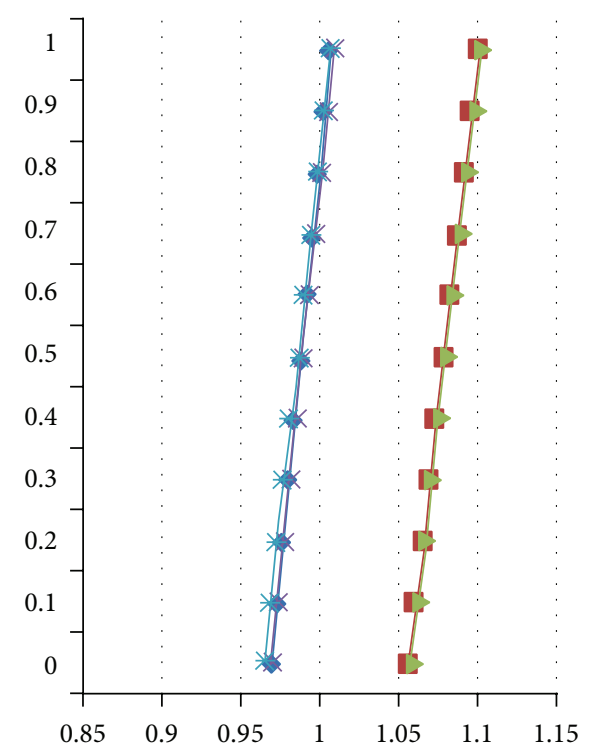

- Lower exact solution for (i)-gH case

- Lower exact solution for (ii)-gH case

$\rightarrow$ Lower numerical solution for step length 0.1

$*$ Lower numerical solution for step length 0.01

* Lower numerical solution for step length 0.001

FIgURE 1: Comparison of lower exact solutions and numerical solution for different step lengths at $t=0.1$.

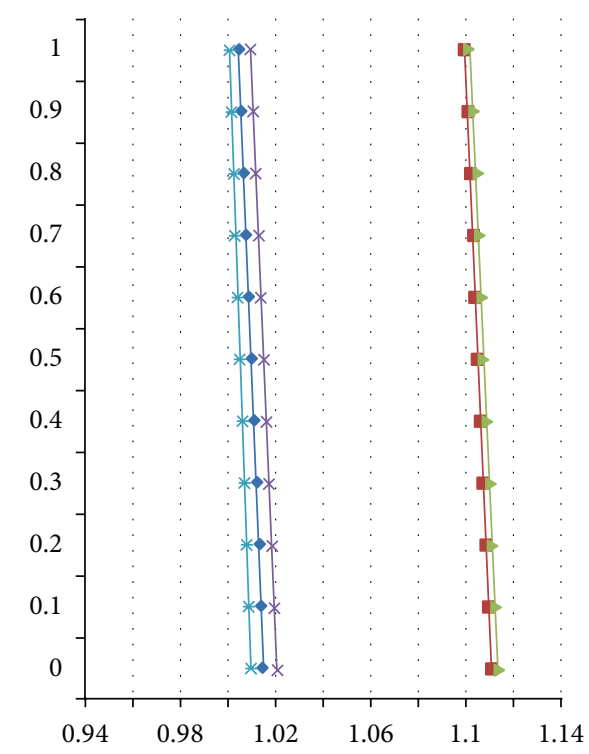

- Upper exact solution for (i)-gH case

$\rightarrow$ Upper exact solution for (ii)-gH case

$\rightarrow$ Upper numerical solution for step length 0.1

$\rightarrow$ Upper numerical solution for step length 0.01

* Upper numerical solution for step length 0.001

FIGURE 2: Comparison of upper exact solutions and numerical solution for different step lengths at $t=0.1$. 


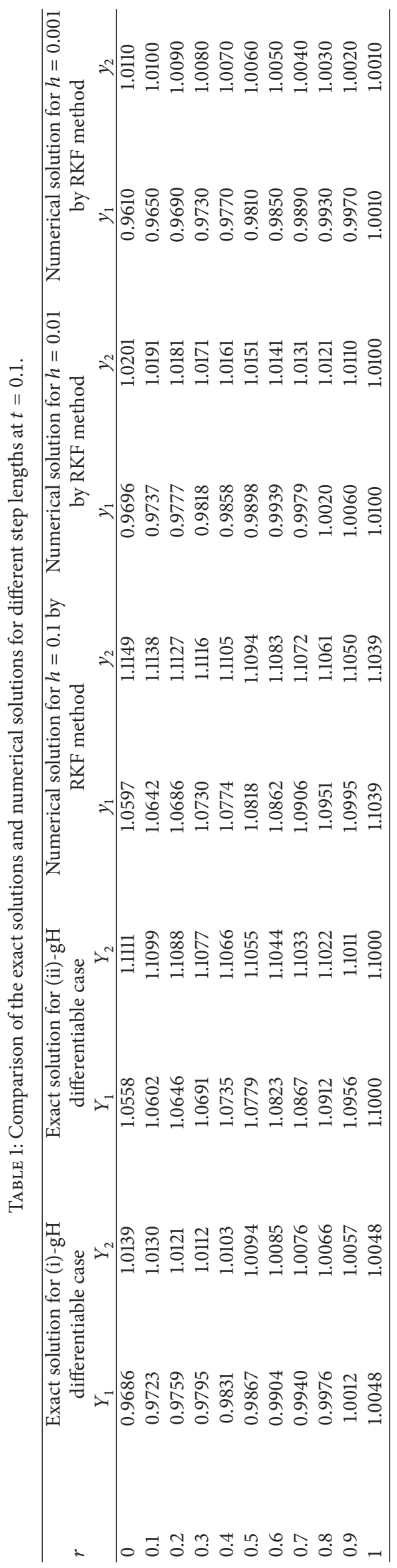




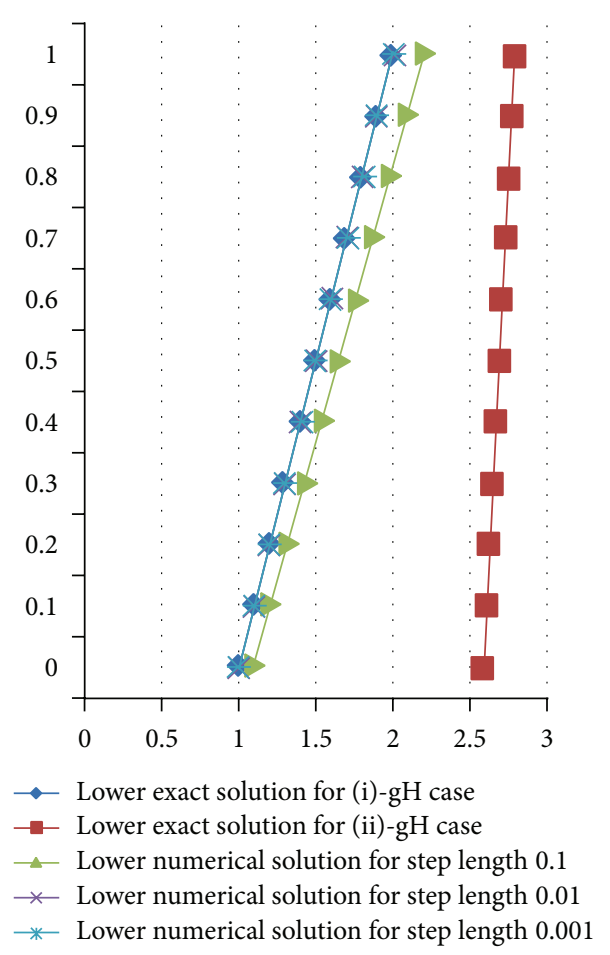

FIGURE 3: Comparison of lower exact solutions and numerical solution for different step lengths at $t=0.4$.

is brine with concentration $k$ lbs salt/gals and the well stirred mixture leaves at the rate 3 gals/min. Let $y(x)$ lbs be the salt in the tank at any time $t \geq 0$. Then $d y(x) / d x+(1 / 100) y(x)=k$, $x \in[0,0.5]$ with $y(0)=c$, if the initial condition is being modeled as fuzzy numbers $c=(1,2,3)$ and $k=(1,2,4)$. Find solution at $x=0.4$.

Solution. For (i)-gH differentiable case the exact solution is

$$
\begin{aligned}
& y_{1}(x, \alpha)=\frac{(1+\alpha)}{100}\left(1+99 e^{-(1 / 100) x}\right), \\
& y_{2}(x, \alpha)=\frac{(2-\alpha)}{50}+\frac{(148-49 \alpha)}{50} e^{-(1 / 100) x} .
\end{aligned}
$$

For (ii)-gH differentiable case the exact solution is

$$
\begin{aligned}
y_{1}(x, \alpha)= & (149-149 \alpha) e^{(1 / 100) x} \\
& +(-248+50 \alpha) e^{-(1 / 100) x} \\
& +(100+100 \alpha), \\
y_{2}(x, \alpha)= & -(149-149 \alpha) e^{(1 / 100) x} \\
& +(-248+50 \alpha) e^{-(1 / 100) x} \\
& +(400-200 \alpha) .
\end{aligned}
$$

Remark 16. From Figure 3 and Table 2 we conclude that the lower exact solution ((i)-gH case) is approximately equal to the numerical solution when we take the step length $h=$ 0.001 (for $h=0.01$ is nearly equal), whereas the lower exact solution ((ii)-gH case) is not equal to any numerical solution.

Remark 17. From Figure 4 and Table 2 we conclude that the upper exact solution ((i)-gH case) is approximately equal to the numerical solution when we take the step length of $h=$ 0.01 and $h=0.001$. For $h=0.1$ it is nearly equal, whereas the upper exact solution ((ii)-gH case) is not equal to any numerical solution.

\section{Conclusion}

In this paper we applied Runge-Kutta-Fehlberg method for finding the numerical solution of first-order linear differential equation in fuzzy environment. The numerical solution is compared with the exact solution ((i)-gH and (ii)-gH both cases). The results presented in the contribution show that Runge-Kutta-Fehlberg method is a powerful mathematical tool for solving first-order linear differential equation in fuzzy environment. The convergence of Runge-KuttaFehlberg method has been discussed. The process method is applied to a mechanical problem in fuzzy environment which shows that it is a promising method to solve the said types of differential equation. In the future we can apply these methods for solving higher order linear and nonlinear differential equation in fuzzy environment. 


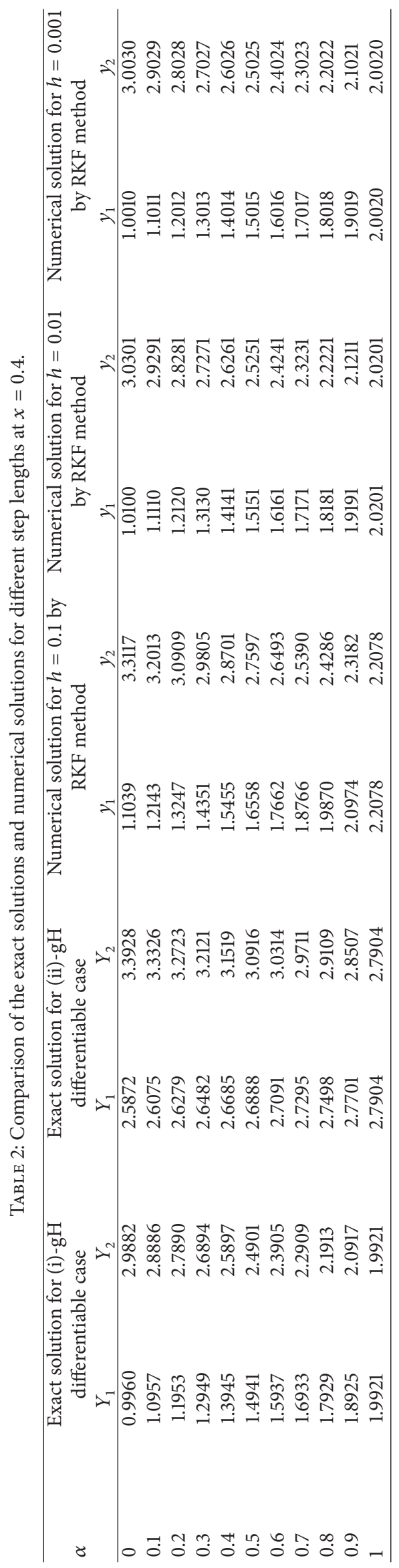




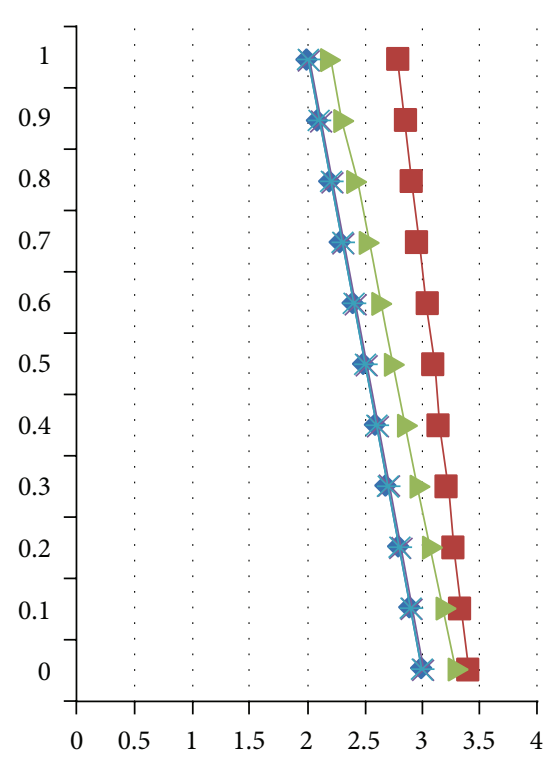

$$
\begin{aligned}
& \rightarrow \text { Upper exact solution for (i)-gH case } \\
& - \text { Upper exact solution for (ii)-gH case } \\
& - \text { Upper numerical solution for step length } 0.1 \\
& * \text { Upper numerical solution for step length } 0.01 \\
& * \quad \text { Upper numerical solution for step length } 0.001
\end{aligned}
$$

FIGURE 4: Comparison of upper exact solutions and numerical solution for different step lengths at $t=0.4$.

\section{Competing Interests}

The authors declare that there are no competing interests.

\section{References}

[1] A. Kandel and W. J. Byatt, "Fuzzy differential equations," in Proceedings of the International Conference Cybernetics and Society, pp. 1213-1216, Tokyo, Japan, 1978.

[2] E. Hüllermeier, "An approach to modelling and simulation of uncertain dynamical systems," International Journal of Uncertainty, Fuzziness and Knowledge-Based Systems, vol. 5, pp. 117137, 1997.

[3] P. Diamond, "Time-dependent differential inclusions, cocycle attractors and fuzzy differential equations," IEEE Transactions on Fuzzy Systems, vol. 7, no. 6, pp. 734-740, 1999.

[4] P. Diamond, "Stability and periodicity in fuzzy differential equations," IEEE Transactions on Fuzzy Systems, vol. 8, no. 5, pp. 583-590, 2000.

[5] P. Diamond and P. Watson, "Regularity of solution sets for differential inclusions quasi-concave in a parameter," Applied Mathematics Letters, vol. 13, no. 1, pp. 31-35, 2000.

[6] B. Bede and S. G. Gal, "Generalizations of the differentiability of fuzzy-number-valued functions with applications to fuzzy differential equations," Fuzzy Sets and Systems, vol. 151, no. 3, pp. 581-599, 2005.

[7] B. Bede, I. J. Rudas, and A. L. Bencsik, "First order linear fuzzy differential equations under generalized differentiability," Information Sciences, vol. 177, no. 7, pp. 1648-1662, 2007.

[8] M. Chen, C. Wu, X. Xue, and G. Liu, "On fuzzy boundary value problems," Information Sciences, vol. 178, no. 7, pp. 1877-1892, 2008.
[9] A. Setia, Y. Liu, and A. S. Vatsala, "Numerical solution of fractional integro-differential equations with nonlocal boundary conditions," Journal of Fractional Calculus and Applications, vol. 5, no. 2, pp. 155-165, 2014.

[10] Y. Liu, "An efficient algorithm for solving differential equations to facilitate modeling and simulation of aerospace systems," SAE Technical Paper 2015-01-2402, 2015.

[11] A. Setia, Y. Liu, and A. S. Vatsala, "Solution of linear fractional Fredholm integro-differential equation by using second kind Chebyshev wavelet," in Proceedings of the 11th International Conference on Information Technology (ITNG '14), pp. 465-469, Las Vegas, Nev, USA, April 2014.

[12] M. Ma, M. Friedman, and A. Kandel, "Numerical solutions of fuzzy differential equations," Fuzzy Sets and Systems, vol. 105, no. 1, pp. 133-138, 1999.

[13] T. Allahviranloo, N. Ahmady, and E. Ahmady, "Two step method for fuzzy differential equations," International Mathematical Forum, vol. 1, no. 17-20, pp. 823-832, 2006.

[14] T. Allahviranloo, N. Ahmady, and E. Ahmady, "Numerical solution of fuzzy differential equations by predictor-corrector method," Information Sciences, vol. 177, no. 7, pp. 1633-1647, 2007.

[15] T. Allahviranloo, E. Ahmady, and N. Ahmady, "Nth-order fuzzy linear differential equations," Information Sciences, vol. 178, pp. 1309-1324, 2008.

[16] S. Pederson and M. Sambandham, "Numerical solution of hybrid fuzzy differential equation IVPs by a characterization theorem," Information Sciences, vol. 179, no. 3, pp. 319-328, 2009.

[17] S. Effati and M. Pakdaman, "Artificial neural network approach for solving fuzzy differential equations," Information Sciences, vol. 180, no. 8, pp. 1434-1457, 2010. 
[18] C. Duraisamy and B. Usha, "Another approach to solution of fuzzy differential equations by modified Euler's method," in Proceedings of the International Conference on Communication and Computational Intelligence (INCOCCI '10), pp. 52-55, Kongu Engineering College, Erode, India, December 2010.

[19] H. Saberi Najafi, F. Ramezani Sasemasi, S. Sabouri Roudkoli, and S. Fazeli Nodehi, "Comparison of two methods for solving fuzzy differential equations based on Euler method and Zadehs extension," The Journal of Mathematics and Computer Science, vol. 2, no. 2, pp. 295-306, 2011.

[20] M. Rostami, M. Kianpour, and E. bashardoust, "A numerical algorithm for solving nonlinear fuzzy differential equations," The Journal of Mathematics and Computer Science, vol. 2, no. 4, pp. 667-671, 2011.

[21] M. Sh. Dahaghin and M. M. Moghadam, "Analysis of a two-step method for numerical solution of fuzzy ordinary differential equations," Italian Journal of Pure and Applied Mathematics, vol. 27, pp. 333-340, 2010.

[22] K. Batiha, "Numerical solutions of the multispecies predatorprey model by variational iteration method," Journal of Computer Science, vol. 3, no. 7, pp. 523-527, 2007.

[23] M. Z. Ahmad and M. K. Hasan, "A new fuzzy version of Euler's method for solving differential equations with fuzzy initial values," Sains Malaysiana, vol. 40, no. 6, pp. 651-657, 2011.

[24] V. Nirmala and S. Chenthur Pandian, "A method to improve the numerical solution of fuzzy initial value problems," International Journal of Mathematical Archive, vol. 3, no. 3, pp. 12311240, 2012.

[25] M. Shafiee, S. Abbasbandy, and T. Allahviranloo, "Predictorcorrector method for nonlinear fuzzy volterra integral equations," Australian Journal of Basic and Applied Sciences, vol. 5, no. 12, pp. 2865-2874, 2011.

[26] M. Ghanbari, "Solution of the first order linear fuzzy differential equations by some reliable methods," Journal of Fuzzy Set Valued Analysis, vol. 2012, Article ID jfsva-00126, 20 pages, 2012.

[27] H. Jafari, M. Saeidy, and D. Baleanu, "The variational iteration method for solving $n$-th order fuzzy differential equations," Central European Journal of Physics, vol. 10, no. 1, pp. 76-85, 2012.

[28] S. Tapaswini and S. Chakraverty, "A new approach to fuzzy initial value problem by improved Euler method," Fuzzy Information and Engineering, vol. 4, no. 3, pp. 293-312, 2012.

[29] M. A. Ahmed and F. S. Fadhel, "Solution of fuzzy initial value problems using Least square and Adomian decomposition method," Journal of Kufa for Mathematics and Computer, vol. 1, no. 4, pp. 49-60, 2011.

[30] R. K. Saikia, "Solution of differential equation by Euler's method using fuzzy concept," International Journal of Computer Technology \& Applications, vol. 3, no. 1, pp. 226-230, 2012.

[31] R. Ezzati, F. Mokhtari, and M. Maghasedi, "Numerical solution of volterra-fredholm integral equations with the help of inverse and direct discrete fuzzy transforms and collocation technique," International Journal of Industrial Mathematics, vol. 4, no. 3, pp. 221-229, 2012.

[32] L. Wang and S. Guo, "Adomian method for second-order fuzzy differential equation," World Academy of Science, Engineering and Technology, vol. 76, pp. 979-982, 2011.

[33] O. S. Fard, "An iterative scheme for the solution of generalized system of linear fuzzy differential equations," World Applied Sciences Journal, vol. 7, no. 12, pp. 1597-1604, 2009.
[34] S. Mehrkanoon, M. Suleiman, and Z. A. Majid, "Block method for numerical solution of fuzzy differential equations," International Mathematical Forum, vol. 4, no. 46, pp. 2269-2280, 2009.

[35] B. Asady and M. Alavi, "Numerical method for solving $n$ thorder linear fuzzy differential equations," Advances in Fuzzy Sets and Systems, vol. 6, no. 2, pp. 95-106, 2010.

[36] C. Duraisamy and B. Usha, "Numerical solution of fuzzy differential equations by Runge-Kutta method of order three," International Journal of Advanced Scientific and Technical Research, vol. 1, no. 1, pp. 1-13, 2011.

[37] V. Nirmala and S. Chenthur Pandian, "Numerical solution of fuzzy differential equation by fourth order Runge-Kutta method with higher order derivative approximations," European Journal of Scientific Research, vol. 62, no. 2, pp. 198-206, 2011.

[38] T. Jayakumar, D. Maheskumar, and K. Kanagarajan, "Numerical solution to fuzzy differential equations by Runge Kutta method of order five," Applied Mathematical Sciences, vol. 6, no. 57-60, pp. 2989-3002, 2012.

[39] B. Ghazanfari and A. Shakerami, "Numerical solutions of fuzzy differential equations by extended Runge-Kutta-like formulae of order 4," Fuzzy Sets and Systems, vol. 189, pp. 74-91, 2012.

[40] K. Kanagarajan and M. Sambath, "Numerical solution of fuzzy differential equations by third order Runge-Kutta method," International Journal of Applied Mathematics and Computation, vol. 2, no. 4, pp. 1-8, 2010.

[41] T. Jayakumar and K. Kanagarajan, "Numerical solution for hybrid fuzzy differential equations by Ruge-Kutta Fehlberg method," International Journal of Mathematical Analysis, vol. 6, no. 53-56, pp. 2619-2632, 2012.

[42] Z. Akbarzadeh Ghanaie and M. Mohseni Moghadam, "Solving fuzzy differential equations by Runge-Kutta method," The Journal of Mathematics and Computer Science, vol. 2, no. 2, pp. 208221, 2011.

[43] M. Ghanbari, "Solution of the first order linear fuzzy differential equations by some reliable methods," Journal of Fuzzy Set Valued Analysis, vol. 2012, Article ID jfsva-00126, 20 pages, 2012.

[44] V. Nirmala and S. Chenthur Pandian, "New multi-step RungeKutta method for solving fuzzy differential equations," Mathematical Theory and Modeling, vol. 1, no. 3, pp. 16-22, 2011.

[45] N. Saveetha and S. Chenthur Pandian, "Numerical solution of fuzzy hybrid differential equation by third order Runge Kutta Nystrom method," Mathematical Theory and Modeling, vol. 2, no. 4, pp. 8-17, 2012.

[46] N. M. Deshmukh, "A new approach to solve Fuzzy differential equation by using third order Runge-Kutta method," ISSN No2031-5063, Vol. 1, Issue 3, pp. 1-4, 2011.

[47] C. Duraisamy and B. Usha, "Numerical solution of fuzzy differential equations by Runge-Kutta method of order four," European Journal of Scientific Research, vol. 67, no. 2, pp. 324337, 2012.

[48] T. Jayakumar and K. Kanagarajan, "Numerical solution for hybrid fuzzy system by Runge Kutta method of order five," Applied Mathematical Sciences, vol. 6, no. 72, pp. 3591-3606, 2012.

[49] J. J. Buckley, T. Feuring, and Y. Hayashi, "Linear systems of first order ordinary differential equations: fuzzy initial conditions," Soft Computing, vol. 6, no. 6, pp. 415-421, 2002.

[50] M. Oberguggenberger and S. Pittschmann, "Differential equations with fuzzy parameters," Mathematical and Computer Modelling of Dynamical Systems, vol. 5, no. 3, pp. 181-202, 1999. 
[51] J. Casasnovas and F. Rossell, "Averaging fuzzy biopolymers," Fuzzy Sets and Systems, vol. 152, no. 1, pp. 139-158, 2005.

[52] M. S. El Naschie, "From experimental quantum optics to quantum gravity via a fuzzy Kähler manifold," Chaos, Solitons \& Fractals, vol. 25, no. 5, pp. 969-977, 2005.

[53] A. Bencsik, B. Bede, J. Tar, and J. Fodor, "Fuzzy differential equations in modeling hydraulic differential servo cylinders," in Proceedings of the 3rd Romanian-Hungarian Joint Symposium on Applied Computational Intelligence (SACI '06), Timisoara, Romania, 2006.

[54] H. Zarei, A. V. Kamyad, and A. A. Heydari, "Fuzzy modeling and control of HIV infection," Computational and Mathematical Methods in Medicine, vol. 2012, Article ID 893474, 17 pages, 2012.

[55] G. L. Diniz, J. F. R. Fernandes, J. F. C. A. Meyer, and L. C. Barros, "A fuzzy Cauchy problem modelling the decay of the biochemical oxygen demand in water," in Proceedings of the Joint 9th IFSA World Congress and 20th NAFIPS International Conference, IEEE, Vancouver, Canada, July 2001.

[56] M. Z. Ahmad and B. De Baets, A Predator-Prey Model with Fuzzy Initial Populations, IFSA-EUSFLAT, 2009.

[57] L. C. Barros, R. C. Bassanezi, and P. A. Tonelli, "Fuzzy modelling in population dynamics," Ecological Modelling, vol. 128, no. 1, pp. 27-33, 2000.

[58] B. Bede, I. J. Rudas, and J. Fodor, "Friction model by using fuzzy differential equations," in Proceedings of the 12th International Fuzzy Systems Association World Congress (IFSA '07), Cancun, Mexico, June 2007, vol. 4529 of Lecture Notes in Computer Science, pp. 23-32, Springer, Berlin, Germany, 2007.

[59] S. P. Mondal, S. Banerjee, and T. K. Roy, "First order linear homogeneous ordinary differential equation in fuzzy environment," International Journal of Pure and Applied Sciences and Technology, vol. 14, no. 1, pp. 16-26, 2013.

[60] S. P. Mondal and T. K. Roy, "First order linear non homogeneous ordinary differential equation in fuzzy environment," Mathematical theory and Modeling, vol. 3, no. 1, pp. 85-95, 2013.

[61] S. P. Mondal and T. K. Roy, "First order linear homogeneous ordinary differential equation in fuzzy environment based on laplace transform," Journal of Fuzzy Set Valued Analysis, vol. 2013, Article ID jfsva-00174, 18 pages, 2013.

[62] S. P. Mondal and T. K. Roy, "First order linear homogeneous fuzzy ordinary differential equation based on lagrange multiplier method," Journal of Soft Computing and Applications, vol. 2013, Article ID jsca-00032, 17 pages, 2013.

[63] S. P. Mondal and T. K. Roy, "First order linear non homogeneous ordinary differential equation in fuzzy environment based on Laplace transform," Journal of Mathematics and Computer Science, vol. 3, no. 6, pp. 1533-1564, 2013.

[64] S. Prasad Mondal and T. Kumar Roy, "First order homogeneous ordinary differential equation with initial value as triangular intuitionistic fuzzy number," Journal of Uncertainty in Mathematics Science, vol. 2014, Article ID jums-00003, 17 pages, 2014.

[65] S. P. Mondal and T. K. Roy, "System of differential equation with initial value as triangular intuitionistic fuzzy number and its application," International Journal of Applied and Computational Mathematics, vol. 1, no. 3, pp. 449-474, 2015.

[66] S. Chakraverty and S. Tapaswini, "Standerd orthogonal polynomial based solution of fuzzy differential equation," The International Journal of Fuzzy Computation and Modelling, vol. 1, no. 1, 2014.

[67] S. Tapaswini and S. Chakraverty, "Numerical solution of fuzzy arbitrary order predator-prey equations," Applications \& Applied Mathematics, vol. 8, no. 2, pp. 647-672, 2013. 


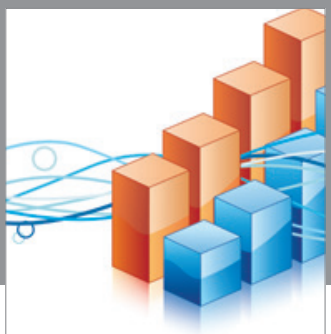

Advances in

Operations Research

vatem alat4

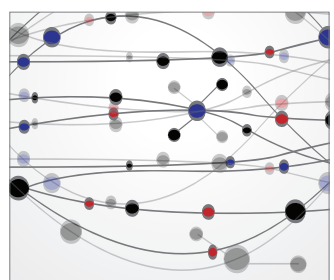

\section{The Scientific} World Journal
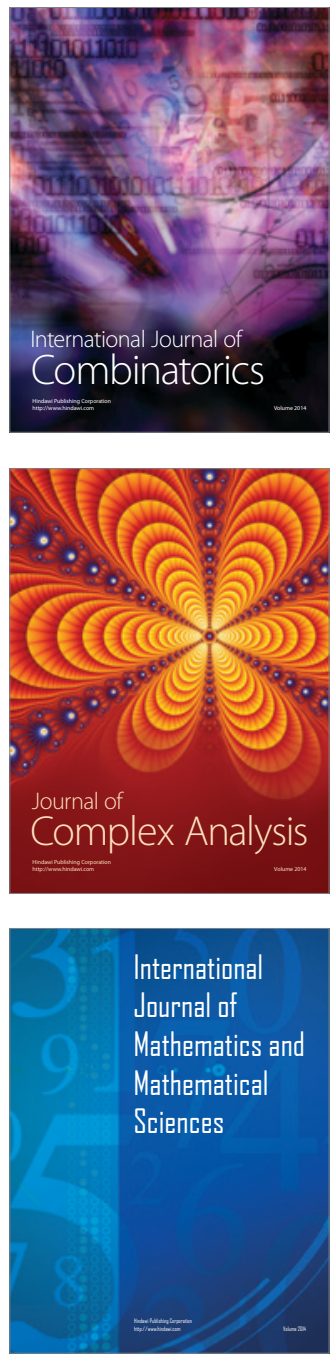
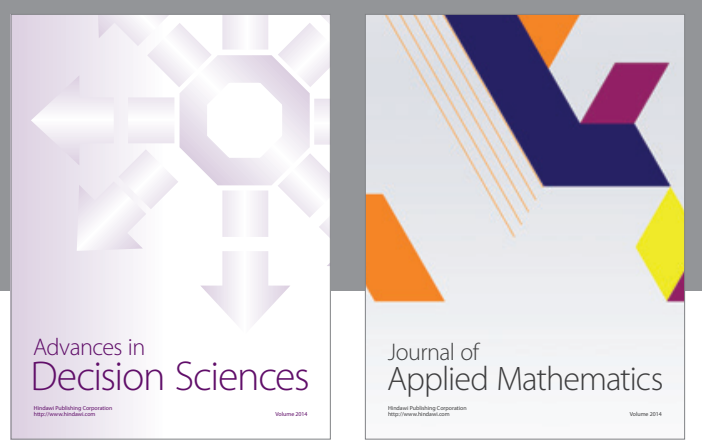

Algebra

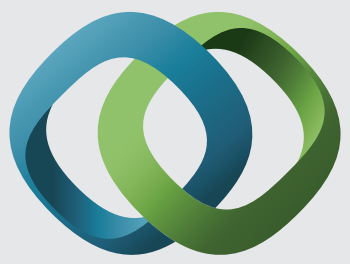

\section{Hindawi}

Submit your manuscripts at

http://www.hindawi.com
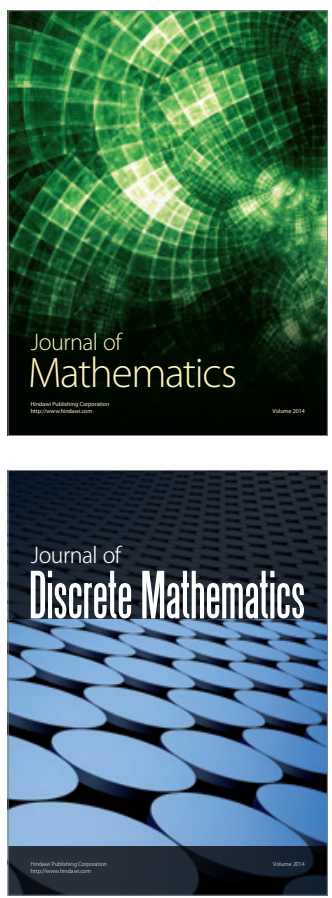

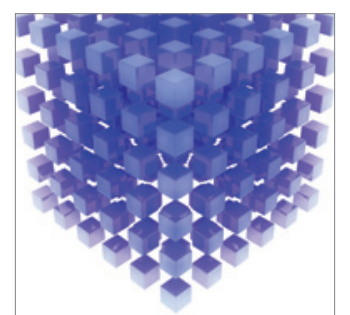

Mathematical Problems in Engineering
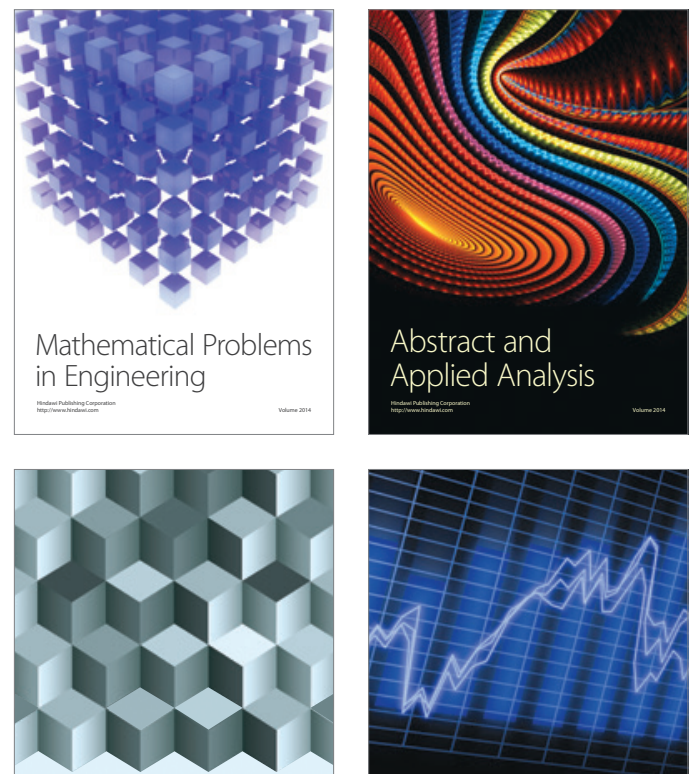

Journal of

Function Spaces

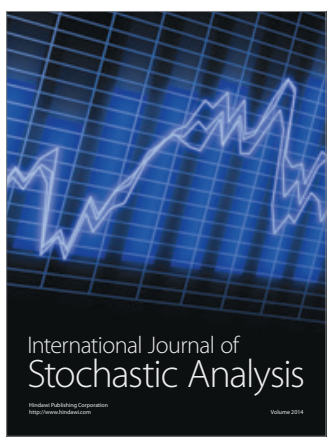

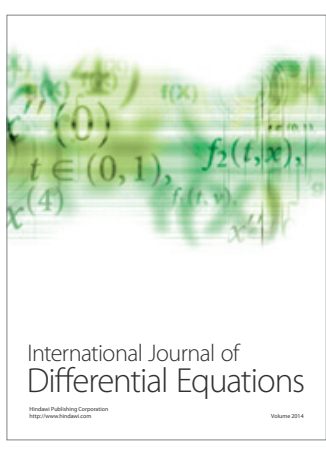
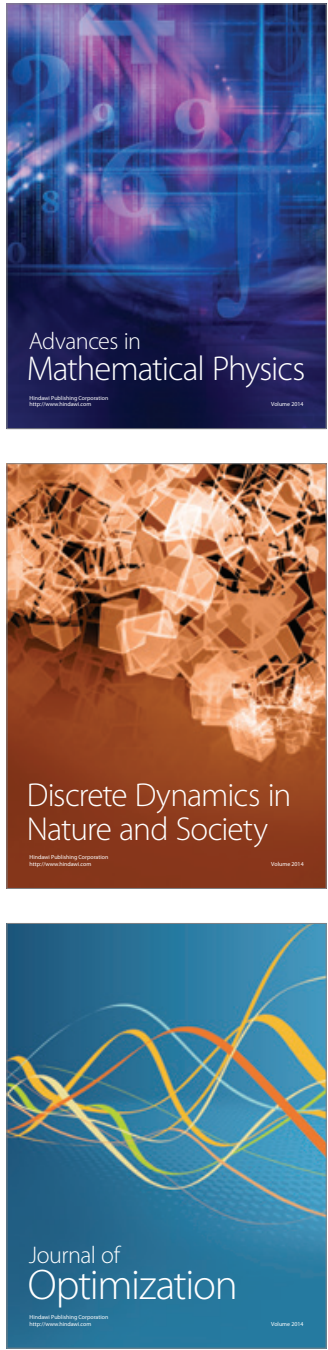